\title{
Oral Cavity as the Seat of Oncohematological Diseases: Extramedullary Plasmacytoma on the Subject of Two Cases
}

\author{
Fabián $\mathrm{PL}^{1 *}$, Cecilia $\mathrm{PC}^{2}$, Gerardo $\mathrm{AM}^{3}$ and Ariel $\mathrm{R}^{3}$ \\ ${ }^{1}$ Consultant in Hematology, National University of La Plata, Argentina \\ ${ }^{2}$ Specialist in Medical Clinic, National University of La Plata, Argentina \\ ${ }^{3}$ Specialist in Surgical Clinic and Head and Neck Surgery, National University of La Plata, \\ Argentina
}

*Corresponding author: Luis Fabián Pintos, Consultant in Hematology, National University of La Plata, Argentina, Tel: +5492214203255; Email: fabianpintos@gmail.com

\section{Case Report \\ Volume 6 Issue 3}

Received Date: August 09, 2021

Published Date: August 19, 2021

DOI: $10.23880 /$ oajds-16000305

\section{Abstract}

Malignant neoplasms of the oral cavity largely (90\%) consist of squamous cell carcinoma arising from the lining mucosa. The remaining $10 \%$ of oral malignancies from a heterogeneous group of tumors of different etiology. We present two cases of oncohematological pathology: Multiple Myeloma

Keywords: Plasmacytoma/pathology; Plasmacytoma/diagnostic; Multiple Myeloma; Mouth Neoplasms/diagnosis

\section{Introduction}

Oral cancer can form anywhere in the mouth. Most oral cancers start in the flat cells that line the surface of your mouth, tongue, and lips. Anyone can get oral cancer, but the risk is higher in men, tobacco users, people with HPV, or a history of head or neck cancer. Frequent sun exposure is also a risk factor for lip cancer. Symptoms of oral cancer include:

a) White or red spots inside the mouth

b) A sore in the mouth that does not heal

c) Hemorrhages in the mouth

d) Loss of teeth

e) Trouble or pain swallowing

f) A lump in the neck

g) Earache

Tests to diagnose oral cancer include a physical exam, endoscopy, biopsy, and imaging tests. Oral cancer treatments can include surgery, radiation therapy, and chemotherapy. Some patients receive a combination of treatments. Frequent dental consultation helps to identify oral cancer in an early stage, giving the patient greater accessibility to adequate treatment, taking into account that no auxiliary diagnostic method replaces a good examination, knowledge of the most frequent changes and the histopathological study such as definitive diagnosis [1-4].

Oral cavity cancer is considered a public health problem because it produces great morbidity and mortality. The most recent estimates from the American Cancer Society for oral cavity and oropharyngeal cancers in the United States for the year 2020 are: About 53,260 people will develop oral cavity and oropharyngeal cancer. In world statistics, oral cavity cancer represents $2.5 \%$ of oncology/oncohematology patients and is responsible for $1 \%$ of cancer deaths [5-8]. Malignant neoplasms of the oral cavity largely $(90 \%)$ consist of squamous cell carcinoma arising from the lining mucosa. The remaining $10 \%$ of oral malignancies from a heterogeneous group of tumors of different etiology, malignant melanoma, malignant tumors intraoral salivary glands, soft tissue sarcomas and jaw bones, non-Hodgkin lymphoma and variants of Multiple Myeloma (oncohematologic disease that corresponds to $1 \%$ of all neoplasms in the USA), and less frequently oral metastases of tumors primary located in other parts of the body. 


\section{Presentation}

Two cases of variants of Multiple Extramedullary Myeloma are presented. Solitary plasmacytoma (there is no diffuse infiltration of the bone marrow) can be bony (half of the cases affect the spine and usually progress to multiple or extraosseous myeloma (affects soft tissues such as the upper respiratory tract and oral mucosa). more frequent in males 3-5: 1 and with a median age of 65 years. Symptoms depend on the location of the tumor, so it can cause airway obstruction, epistaxis, hoarseness, or local pain. 20-30\% present regional lymph node metastases. There are diagnostic criteria for extramedullary plasmacytoma:

a) Extra-bone lesion with infiltration by clonal plasma cells demonstrated by biopsy.

b) Serum monoclonal gammopathy and / or light chains in urine absent or in low concentration.

c) PAMO and MO biopsy without evidence of clonal plasma cells.

d) Absence of lesion in target organs related to MM.

In the diagnostic criteria for bone plasmacytoma, points 2, 3, 4 coincide; while at point 1 a single osteolytic lesion with infiltration by cloned plasma cells is evidenced by biopsy. Lesions can be identified by radiological studies, it is convenient to perform MRI and / or PET-CT to evaluate extension or other locations. Free light chains in serum are useful in its evolutionary control. Surgery and radiation therapy combined are the treatment of choice. The median survival is greater than 10 years.

\section{Case 1}

T.E., male 60 years old.

- AP: HTA, Ex TBQ neck adenocarcinoma: undifferentiated carcinoma of nasopharyngeal origin treated with RT.

- EA: Consult your dentist for loosening of the tooth, seeing a change in color in the maxillary mucosa, so a biopsy is taken.

- Diagnosis: CT: Bilateral maxillary mucosa thickening with bone decrease in the floor of the maxillary sinus. Biopsy: Plasma cell infiltration of the oral mucosa: Clonal neoplasia of plasma cells.

- PAMO and MO Biopsy: normocellular.

- Treatment: local RT.

\section{Case 2}

VS.L., male 57 years old.

AP: No relevant antecedents.

EA: Consultation for a tumor in the right maxillary region.

Diagnosis: MRI: tumor of the right maxillary sinus.

PET-CT: right maxillary sinus formation of soft tissues that generate a lytic process with extension to the ethmoid cells that increase uptake with FDG.

\section{Treatment:}

a. Bisphosphonates: ac. Monthly Zoledronic

b. Local RT

c. Surgery

\section{Case 2 Images}
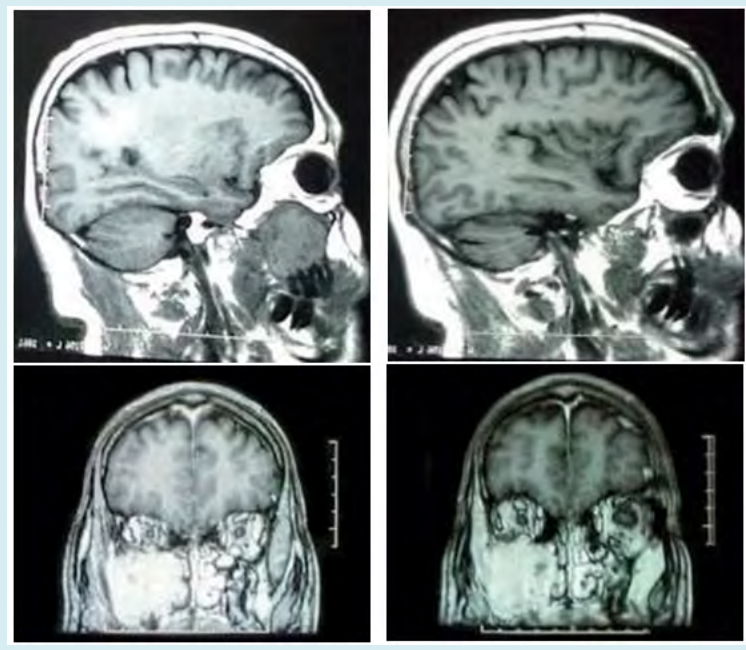

Figures 1-4: MRI of the brain: Presence of a focal image occupying the entire $4.5-\mathrm{cm}$ right maxillary sinus that intensely and slightly heterogeneously enhances after intravenous contrast administration. Bulging of the medial wall that separates it from the nostril as well as from the floor of the orbit. Lysis of the posterior wall of the maxillary sinus. Partial involvement of the lower rectus muscle. 

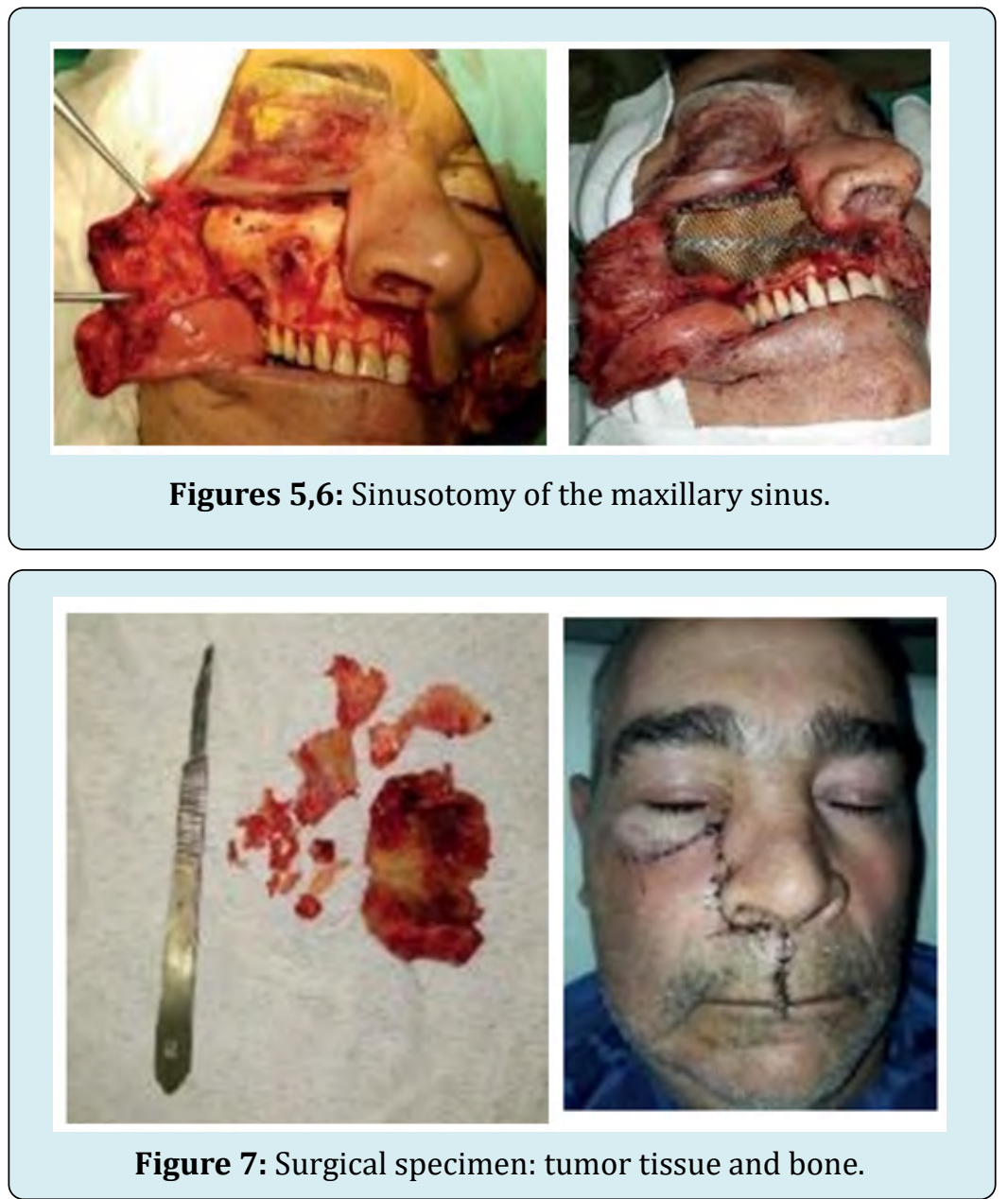

\section{References}

1. Rajkumar SV, Merlini G, San Miguel JF (2012) Haematological cancer. Redefining myeloma. Nat Rev Clin Oncol 9 (9): 494-496.

2. Bladé J (2015) Clinical Monographs in Oncology 13. Chapter 5 Solitary Mileloma and extramedullary plasmacytoma. C. Besses, pp: 59-64.

3. American Cancer Society (2016) Cancer Facts and Figures. Atlanta: American Cancer Society.

4. Pizzolato M (2016) Monoclonal Gammopathies: A methodolical view from the Clinical Laboratory. Hematol 20(1): 99-104.

5. Rajkumar SV (2018) Multiple Myeloma: 2018 update on Diagnosis, Risk-stratification and Management. Am J Hematol 93(8): 981-1114.

6. Argentine Society of Hematology (2019) Diagnostic and Treatment Guidelines. Monoclonal gammopathies.

7. SAH S (2020) Argentine Group of Multiple Myeloma (GAMM-SAH). Hematology, 24.

8. American Cancer Society 2020.

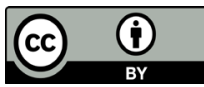

